Für Aerzte, Apotheker und Technologen.

In der Keyserschen Buchhandlung in Erfurt ist erschienen und durch alle Buchhandlungen zu erhalten:

$$
\text { Dr. M. P. Orfila's }
$$

\title{
Handbuch der medizinischen Chemie
}

in Verbindung mit den allgemeinen und technischen Theilen der chemischen Wissenschaft nach ihren neuesten Standpunkte. Aus den Französischen übersetzt von Dr. Fr. T'rommsdorff: Durchigesehen und mit Anmerknngen begleitet von Dr. Jok. Barth.

Trommsd orff. Zwei Bände mit 14 schönen und scharfen Steintafeln. gr. 8.

$$
\text { Preis Rthl. 7. - gr. }
$$

Durch die Uebersetzung dieses vortreffichen Werkes des berihmren Orfila hat unscre Literatur eine dankenswerthe Bereicherung erhalten; denn wenn schon die Recensenten des französischen Originals bemerken, dass solches weder ein angohondor noch ein praktischer Arzt entbehren könne: so gilt woln! dieses um so mebr von der Cebersetzung, de den neuesten Stanipunkt der Wissenschaft umfasst und durch die Bearbeitung dos Herrn Hofrath Trammsdorff und die von Ihnen hinzugefügien Anmerkungen viele Vollständigkeit und Brauchbarkeit gewonnen hat.

In Commission derselben Buchhandlune ist erschienen:

Die Bedingungen und Gesetze des Gleichgewichts; nebst einein Versuche über die Uirsachen der Ruke und Bewegung der hörper. Von Dr. Christian Ernst Meier, ausübendem Arzte in Erfurt, und correspondirenden Mitgliede der naturforschenden Gesellschaft zu Jena. Mit einer Steindrucktafel. Erfurt, 1822. 8. Rthl. 1. oder $1 \mathrm{fl} .3^{6} \mathrm{kr}$.

Die wichtige Lehre vom Gleichgewicht der Körper war bisher ein viel zu wenig bearbeiteter, scliwieriger Gegeistaud der Naturleh. $r e$, “s das man die Bemuhhungen des Herru Verfassers, die Bedin. 
gungen und Gesetze desselben ansumittein, nicht mit verdientem Beifall aufnehmen sollte. Nicht alleln die Theorie der so nützli. chen Schalwage ist durch diese gehaltvolle Schrift berichtigi und vollatändiger geworden, ondern auch von den Ursachen der Rahe und Bewegung der Körper überhaupt erhält der Leser eine neue, hoffentlich befriedigendaro Ansicht, als man bis hieher davon gehabt hat. Kurz, wir glauben, dieses Buch jedem Verebrer der $\mathrm{Na}$ turlelire, der sich eine genauete Kenntniss jener eber so wichtigen als anziehenden Gegenstănde zu rerschatfen wünscht, mit vollem Rechte empfehlen zu können.

\section{Herabgesizter Preis}

des naturbistorischen Prachtwerkes:

Horae physicae Berolinenses, collectae ex symbolis virorum doctorum: $H$. Linkii, C. A. Rudol phi, W. F. Klugii, C. G. Neesii ab Esenbeck, Fr. Ottonis, A. a Chamisso, Fr. Hornschuchii, D. a Schlechtendal et C. G. Ehrenbergii, edi curavit Dr. C. G. Nees ab Esenbeck, cum tabulis aeneis XXVII. gr. fol. 1820. Bonnae apud Marcus. - Früherer Preis Rthl. 12. 12 gr.

Herabgesetzter Preis Rthl. $6.16 \mathrm{gr}$.

Mehrfachen Wunschen zu genägen bietet der Verleger eine Anzahl Exemplare zu dem obigen Preise an, für welchen das Werk bis Ostorn 1823 durch alle Buchbandlungen zu erhalten ist. -

Später tritt der Ladenpreis bestimmt wieder ein, so wie auch früher sehon, wenn die beatimmte Anzahl Exemplare etwa früher sehon vergriffon seyn solite.

Auch können Exemplare mit schwarzen Kopfern zu Rthl. 4 . besorgt werden. 